\title{
New Induction Heating Coils with Reduced Iron-Loss in the Cores for Small-Foreign-Metal Particle Detector Using an SiC-MOSFETs High-Frequency Inverter
}

\author{
Takuya Shijo* \\ Student Member, \\ Yuki Uchino* \\ Student Member \\ Yujiro Noda* \\ Member, \\ Hiroaki Yamada* \\ Member \\ Toshihiko Tanaka ${ }^{* a)}$ Fellow
}

(Manuscript received July 20, 2018, revised April 24, 2019)

\begin{abstract}
This paper presents iron loss reduction in the cores of induction heating (IH) coils for a small-foreign-metal particle (SFMP) detector with a 400-kHz SiC-MOSFETs high-frequency inverter. A new core shape for IH coils, which can reduce iron loss, is designed for the stable and continuous operation of the SFMP detector. Magnetic field analysis results using JSOL JMAG software, a 3D full-wave electromagnetic field simulation software, demonstrate that the iron loss caused by the new core shape for IH coils is decreased by $71.8 \%$, compared with that of the previously proposed IH coils, which increase the magnetic flux density to heat SFMPs by $20 \%$. Thus, the stable and continuous operation of the SFMP detector with the newly designed core shape of IH coils can be achieved. It is also shown that SFMP represented by $0.3-\mathrm{mm}$ diameter stainless-steel balls (SUS304) can be detected on the high-performance chemical film (HPCF) with the newly designed core shape of IH coils using JSOL JMAG software. A prototype experimental setup of the SFMP detector with the new core shape of IH coils is constructed and tested. Experimental results demonstrate that a 400-kHz SiC-MOSFETs high-frequency-inverter-based SFMP detector with the newly designed core shape of IH coils can heat SFMPs, which can be observed by a thermographic camera. Therefore, the authors conclude that the 400-kHz SiC-MOSFETs high-frequency-inverter-based SFMPs detector with the newly designed core shape of IH coils is applicable for HPCF production lines.
\end{abstract}

Keywords: SiC-MOSFETs, small-foreign-metal particle detector, induction heating, iron loss reduction

\section{Introduction}

High-performance chemical films (HPCFs) are widely used in lithium-ion batteries (LiBs) and flat panel displays of notebook computers, notepads, and mobile phones. For example, they are used as the separators of LiBs. The film material is plastic, and the required thickness is less than $0.01 \mathrm{~mm}$. However, during the manufacturing process of the HPCFs, small-foreign-metal particles (SFMPs) produced by the manufacturing machines occasionally adhered to them. The SFMPs adhering to the HPCFs are typically made up of stainless steel or iron, and their diameter is less than approximately $1.0 \mathrm{~mm}$. It is well known that SFMPs adhering to HPCFs rarely construct a short circuit between the positive electrode and negative electrode through the separator, which comprising the HPCFs. Overheating in the LiBs occurs due to this short circuit ${ }^{(1)(2)}$. Therefore, there is a strong requirement in industry for practical methods of detecting SFMPs.

Ikeda et al. proposed a small-conductive-particles detection using a microwave resonant cavity ${ }^{(3)}$. It was shown

a) Correspondence to: Toshihiko Tanaka. E-mail: totanaka@ yamaguchi-u.ac.jp

* Department of Electrical and Electronic Engineering, Yamaguchi University

2-16-1, Tokiwadai, Ube, Yamaguchi 755-8611, Japan experimentally that minimum-detectable diameter of sphere particles is approximately $0.05 \mathrm{~mm}$. However, the smallforeign-metal particles adhered to HPCFs were inspected in the slit in a microwave cavity, where the slit size was $2 \times 70 \mathrm{~mm}$. In the continuous production lines of HPCF, HPCFs are going through in the slit. A longer slit is required for the actual-continuous production lines of HPCFs. Therefore, the practicability of the proposed small-conductiveparticle detection method is unclear. Nakai et al. proposed a detection method for a small-magnetic-particles with a sensitive thin-film sensor ${ }^{(4)}$. However, a high magnetic flux density is required in this detection method. For the practical use of a small-magnetic-particles detector, the power sources, which can generate the high magnetic flux for the sensitive thin-film sensor is strongly required. Tanaka et al. proposed an SFMP detection method with a high-temperature scanning superconductive quantum interference device (HTS SQUID) gradiometer system ${ }^{(5)-(8)}$. They demonstrated that small iron particles with a diameter of less than $0.1 \mathrm{~mm}$ can be detected with the HTS SQUID. However, the SQUID needs a highly reliable cooling system to achieve superconductivity. Thus, a more practicable SFMP detector is required for industrial applications. The present authors proposed an IH-based SFMPs detection method with a high-frequency inverter ${ }^{(9)-(12)}$. In (9), the winding locations on the core of the induction heating 


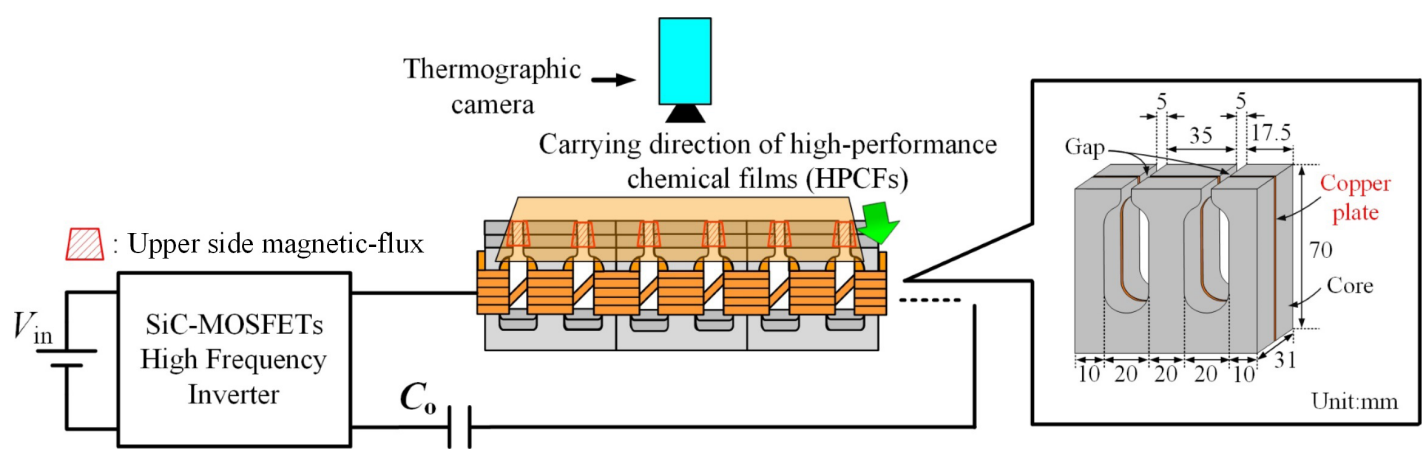

Fig. 1. Block diagram and core dimensions of the previously proposed small-foreign-metal particle (SFMP) detector using SiC-MOSFETs high-frequency inverter

(IH) coils were discussed in detail. The magnetic field analysis results with JSOL JMAG software, a 3D full-wave electromagnetic field simulation software, demonstrated that placing the windings on the core close to the gap is effective in reducing iron loss in the core of the IH coils. The validity and high practicability of the IH-based SFMP detector with the new winding locations on the IH coils were experimentally confirmed ${ }^{(10)}$. Experimental results demonstrated that a $0.15-\mathrm{mm}$ diameter SFMP can be heated using a $400-$ $\mathrm{kHz}$ high-frequency inverter with SiC-MOSFETs and observed with a thermographic camera. However, this proposed IH-based SFMP detection method with a high-frequency inverter cannot be applied to actual continuous HPCF production lines because the SFMP was located in the gap in the core of the IH coils. The present authors also proposed a detection method using a magnetic flux outside core gap ${ }^{(11)(12)}$. Figure 1 shows a block diagram of the previously proposed SFMP detector using the SiC-MOSFETs high-frequency inverter. In (11), an upper side magnetic-flux of the core gap of $30 \mathrm{mT}$ was used to heat and detect $0.3-\mathrm{mm}$ diameter SFMPs. The IH coils generate a high-frequency magnetic flux, which induces eddy currents in the SFMPs. The induced eddy current raises the temperature of the SFMPs. Subsequently, the heated SFMPs expand their own thermal energy heat into HPCFs heat diffusion, and they are thus easily observed with a thermographic camera. However, core cracking of the previously proposed IH coils in Fig. 1 often occurred owing to thermal stress caused by the iron losses. Therefore, the SFMP detector with the previously proposed IH coils in Fig. 1 cannot achieve the stable and continuous operation that is desired in an HPCF production line.

In this paper, a core shape of $\mathrm{IH}$ coils with a $400-\mathrm{kHz}$ SiC-MOSFETs high-frequency-inverter-based SFMP detector is newly designed for iron loss reduction. A magnetic field analysis is performed to confirm the validity and high practicability of the newly designed core shape of IH coils using JSOL JMAG software. Magnetic field analysis results demonstrate that the iron loss in the new IH core is reduced by $71.8 \%$, compared with that in a previously proposed IH core increasing the magnetic flux density to heat the SFMPs by $20 \%$. Reducing the iron loss can avoid the temperature rise in the newly designed core of IH coils. This means that core cracking, which often occurred in the literature (11) and(12), can also be avoided. A prototype experimental model of the high-frequency SiC-MOSFETs based SFMP detector with the newly designed core shape in $\mathrm{IH}$ coils is constructed and tested. Experimental results demonstrate that a 400-kHz SiC-MOSFETs high-frequencyinverter-based SFMP detector with the newly designed core shape in IH coils can heat $0.3-\mathrm{mm}$ diameter SFMPs, and the heated SFMPs can be observed by a thermographic camera. From the experimental results, the authors conclude that the 400-kHz SiC-MOSFETs high-frequency inverter-based SFMPs detector with the newly designed core shape in IH coils is applicable for the practical production line of HPCFs.

\section{Newly Designed Core Shape of IH Coils for Iron Loss Reduction}

Figure 2 shows a block diagram and core dimensions of the SFMP detector with the newly designed core shape of $\mathrm{IH}$ coils. A $400-\mathrm{kHz} \mathrm{SiC}-\mathrm{MOSFETs}$ high-frequency inverter is also used with the newly designed core shape of IH coils to generate high-frequency magnetic flux. The newly designed core shape of IH coils comprising of an E-shaped core, an Ishaped core, and a copper plate, with a high-frequency magnetic flux generated in the core gap between the E-shaped core and I-shaped core. The HPCFs pass through the gap. The gap length between the E-shaped core and I-shaped core is $5 \mathrm{~mm}$. A Litz wire can be wound around in the E-shaped core. Therefore, iron loss can be reduced. The E-shaped and I-shaped cores shown in Fig. 2 are connected in multiple series and parallel, respectively. In (11) and (12), the frequent occurrence of core cracking was reported, caused by temperature differences in the cores. To sink the temperature difference in the cores, a copper plate of 1-mm-thickness is inserted in the cores, effectively avoiding core cracking. The turn-number of windings for each core leg is five turns. Just manufactured HPCFs are suspended at a height of $3 \mathrm{~mm}$ from the upper-surface of the E-shaped cores. As the IH coil produces a high-frequency magnetic field in the core gap between the I-shaped and E-shaped cores, the SFMPs on the film are heated and then observed using a thermographic camera. The number of multiple-series-parallel connections of IH coils depends on the width of the manufactured HPCFs.

The switching frequency of the SiC-MOSFETs inverter has been discussed in (10). The relationships between the eddy-current Joule losses $P_{\mathrm{E}} \mathrm{W}$ and the alternating magnetic flux frequency $f \mathrm{~Hz}$ along with the magnetic flux density $B \mathrm{~T}$ can be expressed as 


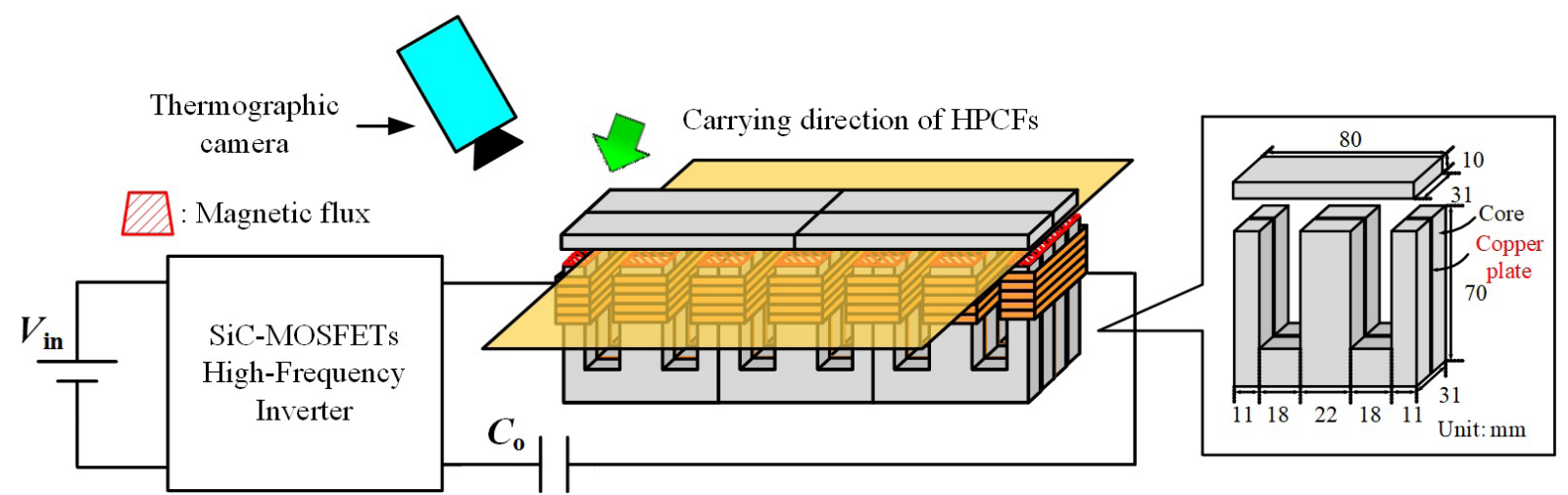

Fig. 2. Block diagram and core dimension of the SFMP detector using newly designed core shape of induction heating (IH) coils

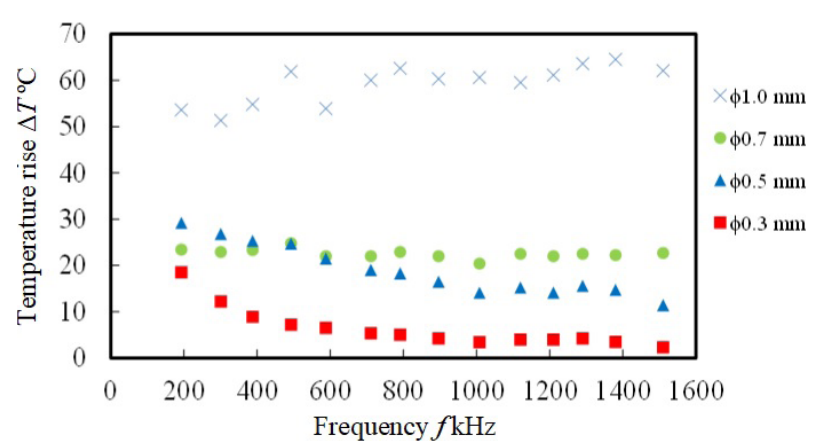

Fig. 3. Measured relationship between $\Delta T$ and frequency

$$
P_{\mathrm{E}} \propto(B \cdot f)^{2}
$$

Equation (1) shows that the Joule losses $P_{\mathrm{E}}$ generated in the SFMPs are proportional to the square of $B \cdot f$. The frequency and the magnetic flux density to heat SFMPs, SUS304, are discussed experimentally using (1). Figure 3 shows the measured relationship between the frequency of the magnetic flux and the temperature rise $\Delta T$ of the small metal particles. In Fig. 3, frequencies of magnetic flux density are ranged from $200 \mathrm{kHz}$ to $1300 \mathrm{kHz}$ with $5 \mathrm{sec}$ radiation. The coil current was varied to achieve the approximately constant $B \cdot f$, where $B \cdot f$ is approximately unity. The temperature rise $\Delta T$ is almost constant for $1.0-\mathrm{mm}$ and 0.7 mm diameter particles; these experimental results satisfy (1). However, temperature rise $\Delta T$ decreases with the higher frequency for 0.5 - $\mathrm{mm}$ and 0.3 -mm diameter particles; these experimental results disagree with (1). Thus, Fig. 3 demonstrates that lower frequencies of the flux density is enough in heating $0.5-\mathrm{mm}$ and $0.3-\mathrm{mm}$ diameter particles. In Japan, the frequencies in the range of $526.5-1606.6 \mathrm{kHz}$ are used in amplitude modulation (AM) radio broadcasting. The use of the frequency above $500 \mathrm{kHz}$ is prohibited by Japanese law. It is also well known that lower frequencies increase the capacitance of the resonant capacitors. From Fig. 3 and Japanese law, the switching frequency of SiC-MOSFETs inverter has been set to $400 \mathrm{kHz}$ in this paper.

Figure 4 shows the magnetic flux path of the series connected IH cores as shown in Fig. 1. The purpose of this paper is to heat and observe SFMPs, which are the $0.3-\mathrm{mm}$ diameter stainless balls (SUS304). The magnetic flux density of approximately $36 \mathrm{mT}$ is required to heat the $0.3-\mathrm{mm}$ diameter stainless balls in the experimental results of Section 3. In

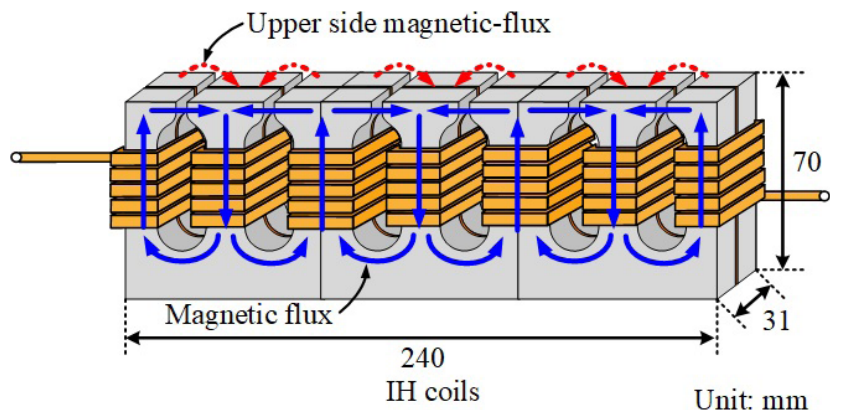

Fig. 4. Magnetic flux path of series connected IH cores shown in Fig. 1

Fig. 4, an upper side magnetic flux is used to heat SFMPs. The upper side magnetic flux density is lower than the magnetic flux density in the core gap between E-shaped and Ishaped cores as shown in Fig. 2. This means that the larger coil-current in Fig. 4 is required than that in Fig. 2 to generate higher magnetic flux. As well known, the larger coil currents cause the iron loss in the core, which consists of Joule loss and Hysteresis losses. This larger iron loss often results in core cracking in the previously proposed IH cores (11). The newly proposed IH coils with E-shaped and I-shaped cores in Fig. 2, which can reduce the iron loss in cores, are more applicable for the practical HPCFs production lines.

Figure 5 shows the magnetic flux path in the previously proposed core and its magnetic equivalent circuit. Figure 5(a) shows the magnetic flux path in the previously proposed core. Figure 5(b) shows the magnetic equivalent circuits of Fig. 5(a). In Fig. 5(b), $\phi_{1}, \phi_{2}$, and $\phi_{3}$ is the magnetic flux in the core and air gap respectively. $R_{1}, R_{2}$, and $R_{\mathrm{g}}$ are the magnetic resistance of each magnetic path length respectively. $l_{1}$, $l_{2}, l_{3}$, and $l_{\mathrm{g}}$ are the magnetic path length of each core and core gap. $S_{1}, S_{2}$, and $S_{\mathrm{g}}$ are the cross-sectional area of each core. The magnetic resistance of each magnetic path are expressed as

$$
\begin{aligned}
& R_{1}=\frac{l_{1}}{\mu_{0} \mu_{S} S_{1}}, \\
& R_{2}=\frac{\left(l_{2}+l_{3}\right)}{\mu_{0} \mu_{S} S_{2}}, \\
& R_{\mathrm{g}}=\frac{l_{\mathrm{g}}}{\mu_{0} S_{\mathrm{g}}}, \cdots
\end{aligned}
$$

where the magnetic path length $l_{1}, l_{2}, l_{3}$, and $l_{\mathrm{g}}$ are $35 \mathrm{~mm}$, 


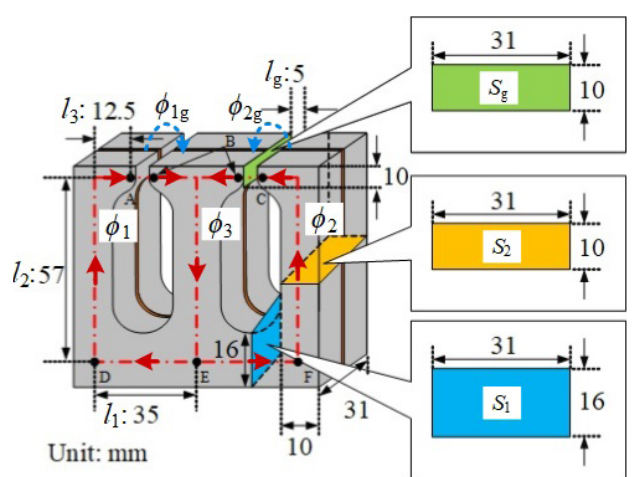

(a)

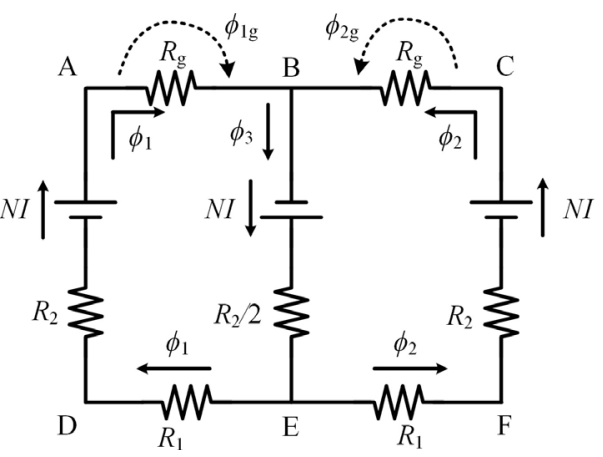

(b)

Fig. 5. Magnetic flux path in previously proposed IH core and its magnetic equivalent circuit. (a) Magnetic flux path in previously proposed IH core. (b) Magnetic equivalent circuit of Fig. 5(a)

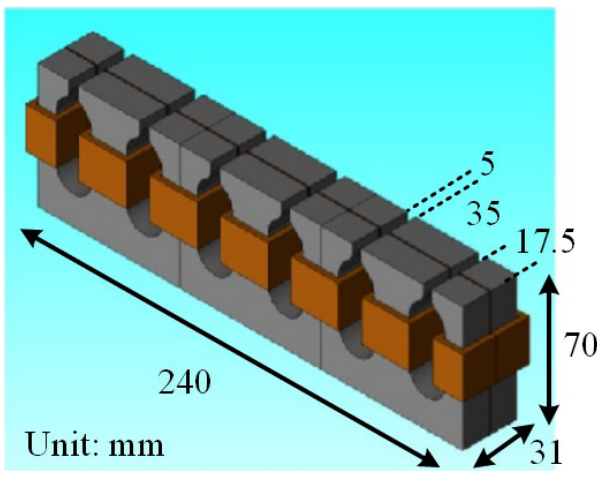

(a)

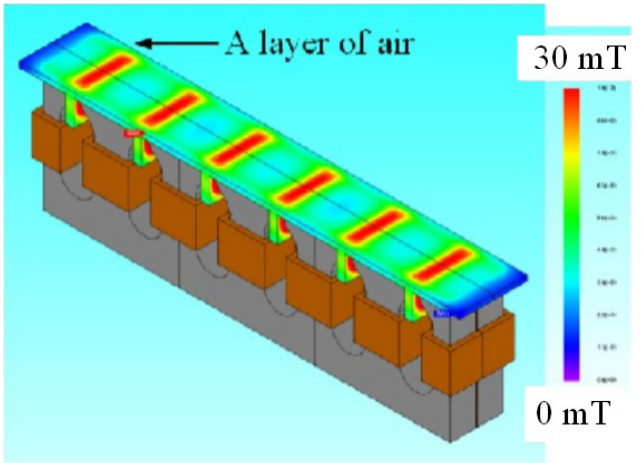

(b)

Fig. 6. Magnetic field analysis results of the magnetic flux density near the core gap modeled with JMAG software. (a) FEM model. (b) Magnetic field analysis result

$57 \mathrm{~mm}, 12.5 \mathrm{~mm}$, and $5 \mathrm{~mm}$ respectively. The cross-sectional area of each core $S_{1}, S_{2}$, and $S_{\mathrm{g}}$ are $31 \times 16 \mathrm{~mm}^{2}$, $31 \times 10 \mathrm{~mm}^{2}$, and $31 \times 10 \mathrm{~mm}^{2}$. The magnetic permeability $\mu_{0}$ of vacuum is $4 \pi \times 10^{-7} \mathrm{H} / \mathrm{m}$. The relative permeability $\mu_{\mathrm{S}}$ of IH core is 2400 .

From Kirchhoff's law in magnetic circuit, the magnetic flux $\phi_{1}, \phi_{2}$, and $\phi_{3}$ are expressed as

$$
\begin{aligned}
& \phi_{1}+\phi_{2}=\phi_{3}, \cdots \cdots \cdots \cdots \cdots \cdots \cdots \\
& \frac{R_{2}}{2} \phi_{3}+\left(R_{1}+R_{2}+R_{\mathrm{g}}\right) \phi_{1}=2 N I, \\
& \frac{R_{2}}{2} \phi_{3}+\left(R_{1}+R_{2}+R_{\mathrm{g}}\right) \phi_{2}=2 N I, \cdots
\end{aligned}
$$

where turn-number $N$ is 5 , and coil current $I$ is $30 \mathrm{Arms}$, respectively. The magnetic resistance $R_{1}, R_{2}$, and $R_{\mathrm{g}}$ of each magnetic path length are $23 \mathrm{H}^{-1}, 74 \mathrm{H}^{-1}$, and $12835 \mathrm{H}^{-1}$ respectively. In (5)-(7), calculation results of magnetic flux density in the core gap is $75.1 \mathrm{mT}$ with the magnetic flux $\phi_{1}$. However, the magnetic flux $\phi_{1 \mathrm{~g}}$ for heating of SFMPs, which is at $3 \mathrm{~mm}$ upper side from the surface of the E-shaped core cannot be calculated by Fig. 5(b). Thus, the magnetic flux density $\phi_{1 \mathrm{~g}}$ is calculated using JSOL JMAG software. Figure 6 shows the calculated magnetic flux density near the core gap of previously designed E-shaped core of Fig. 4. Figure 6(a) shows a finite element method (FEM) model, and Fig. 6(b) shows the magnetic field analysis results of the magnetic flux density distribution at $3 \mathrm{~mm}$ upper side from the surface of the E-shaped core, where the core material is PC40 (TDK Co., Ltd.). As shown in Fig. 6(b), the magnetic

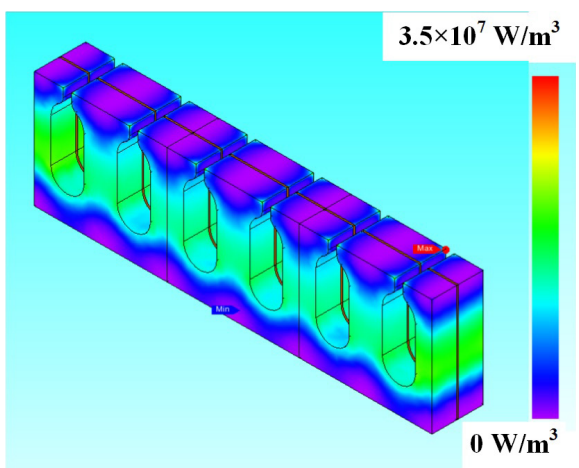

Fig. 7. Iron loss density distribution in previously proposed IH cores using JSOL JMAG software

flux density near the core gap is approximately $30 \mathrm{mT}$ with the coil current of 30 Arms. Figure 7 shows the iron loss density distribution in the previously proposed IH cores of Fig. 4. In Fig. 7, the maximum iron loss density is $3.15 \times 10^{7} \mathrm{~W} / \mathrm{m}^{3}$. This higher loss density often caused core cracking. Thus, the previously proposed IH coils is not suitable for the practical use of SFMPs detector.

Figure 8 shows the magnetic flux path of series connected IH cores, which was shown in Fig. 2. Figure 9 shows the magnetic flux path in the newly proposed IH core and its magnetic equivalent circuit. Figure 9(a) shows the magnetic flux path in the newly proposed IH core. Figure 9 (b) shows the magnetic equivalent circuits of Fig. 9(a). In Fig. 9(b), $\phi_{1}$, $\phi_{2}$, and $\phi_{3}$ are the magnetic flux in the core and air gap, 
respectively. $R_{1}, R_{2}, R_{3}$, and $R_{\mathrm{g}}$ are the magnetic resistance of each magnetic path, respectively. $l_{1}, l_{2}, l_{3}$, and $l_{\mathrm{g}}$ are the magnetic path length. $S_{1}, S_{2}, S_{3}$, and $S_{\mathrm{g}}$ are the cross-sectional area of each path. The magnetic resistance of each magnetic path are expressed as

$$
\begin{aligned}
& R_{1}=\frac{l_{1}}{\mu_{0} \mu_{S} S_{1}}, \\
& R_{2}=\frac{l_{2}}{\mu_{0} \mu_{S} S_{2}}, \\
& R_{3}=\frac{l_{3}}{\mu_{0} \mu_{S} S_{3}}, \\
& R_{\mathrm{g}}=\frac{l_{\mathrm{g}}}{\mu_{0} S_{\mathrm{g}}}, \cdots
\end{aligned}
$$

where the magnetic path length $l_{1}, l_{2}, l_{3}$, and $l_{\mathrm{g}}$ are $35 \mathrm{~mm}$, $61 \mathrm{~mm}, 35 \mathrm{~mm}$, and $5 \mathrm{~mm}$, respectively. The cross sectional area of each core $S_{1}, S_{2}, S_{3}$, and $S_{\mathrm{g}}$ are $31 \times 18 \mathrm{~mm}^{2}$, $31 \times 11 \mathrm{~mm}^{2}, 31 \times 11 \mathrm{~mm}^{2}$, and $31 \times 10 \mathrm{~mm}^{2}$ respectively. The magnetic permeability $\mu_{0}$ of vacuum is $4 \pi \times 10^{-7} \mathrm{H} / \mathrm{m}$. The relative permeability $\mu_{\mathrm{S}}$ of IH core is 2400 .

From Kirchhoff's law in the magnetic circuits, the magnetic flux $\phi_{1}, \phi_{2}$, and $\phi_{3}$ are expressed as

$$
\begin{aligned}
& \phi_{1}+\phi_{2}=\phi_{3}, \cdots \cdots \cdots \cdots \cdots \cdots \cdots \cdots \cdots \\
& \left(\frac{R_{\mathrm{g}}}{2}+\frac{R_{2}}{2}\right) \phi_{3}+\left(R_{1}+R_{2}+R_{3}+R_{\mathrm{g}}\right) \phi_{1}=2 N I, \\
& \left(\frac{R_{\mathrm{g}}}{2}+\frac{R_{2}}{2}\right) \phi_{3}+\left(R_{1}+R_{2}+R_{3}+R_{\mathrm{g}}\right) \phi_{2}=2 N I,
\end{aligned}
$$

where the turn-number $N$ is 5 , and the coil current $I$ is 30 Arms, respectively. The magnetic resistance of each magnetic path length $R_{1}, R_{2}, R_{3}$, and $R_{\mathrm{g}}$ are $21 \mathrm{H}^{-1}, 59 \mathrm{H}^{-1}$,

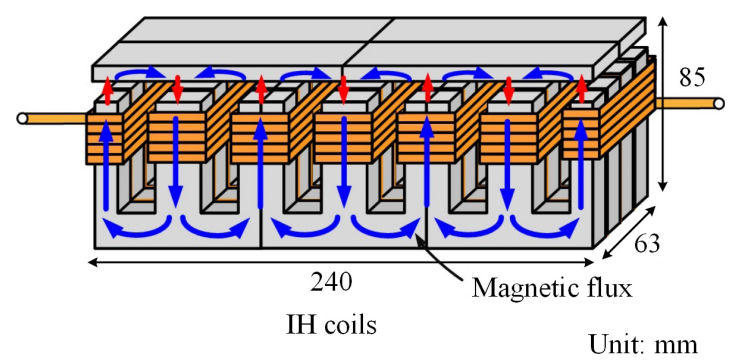

Fig. 8. Magnetic flux path of series connected IH cores shown in Fig. 2

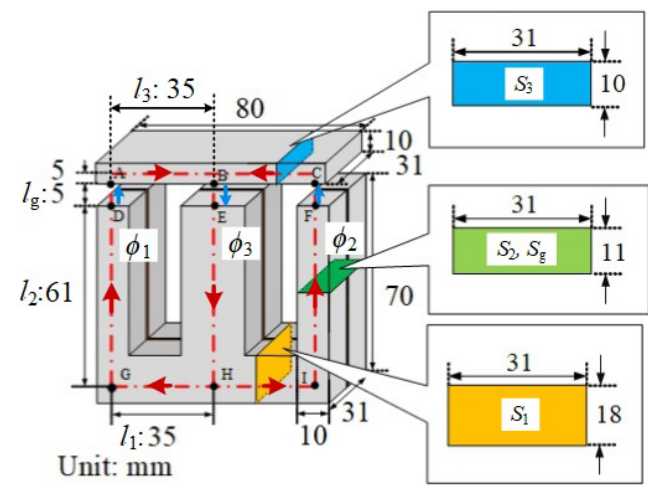

(a)

Fig. 9. Magnetic flux path in newly proposed IH core and its magnetic equivalent circuit. (a) Magnetic flux path in newly proposed IH core. (b) Magnetic equivalent circuit of Fig. 9(a)
$39 \mathrm{H}^{-1}$, and $11668 \mathrm{H}^{-1}$, respectively. In (12)-(14), calculation results of magnetic flux density in the core gap is $37.5 \mathrm{mT}$. Magnetic field analysis is here performed to confirm the reduction effect of the iron loss in the newly designed core shape of IH coils using JSOL JMAG software. The frequency of the coil current is $400 \mathrm{kHz}$, and its rootmean-square (RMS) value is 30 Arms.

Figure 10 shows the magnetic field analysis results of the newly designed IH core in Figs. 2 and 8 using JSOL JMAG software. Figure 10(a) shows a finite element method (FEM) model, and Fig. 10(b) shows the magnetic field analysis results of the magnetic flux density distribution at $3 \mathrm{~mm}$ from the upper-surface of the newly designed E-shape core, where the core material is PC40 (TDK Co., Ltd.). As shown in Fig. 10(b), the magnetic flux density in the core gap is approximately $36 \mathrm{mT}$ with the coil current of $30 \mathrm{Arms}$. The simulation results of Fig. 9 is well agree with the calculation results from (8)-(14). In Fig. 6(b), the magnetic flux density at $3 \mathrm{~mm}$ upper side from the surface of the E-shaped core was $30 \mathrm{mT}$ with the coil current of 30 Arms. Therefore, the magnetic flux density for heating the SFMPs is increased by $20 \%$ using the newly designed core shapes of IH coils.

Figure 11 shows the iron loss density distribution in the newly proposed IH cores as shown in Fig. 10 using JSOL JMAG software. In Fig. 11, the maximum iron loss density is $2.83 \times 10^{7} \mathrm{~W} / \mathrm{m}^{3}$. Table 1 presents the iron loss comparisons between the previously and newly designed core shapes of IH coils, which are Figs. 2 and 8(b), where three cores are connected in series. It is well known that the iron losses in the cores of IH coils are caused by Joule and hysteresis losses. In the previously proposed cores, the Joule loss is $2686 \mathrm{~W}$, and the hysteresis loss is $1402 \mathrm{~W}$. Thus, the total iron loss is $4088 \mathrm{~W}$ while the total iron loss in the newly designed core shape of IH coils is $1153 \mathrm{~W}$. The iron loss in the newly designed core shape of IH coils is reduced by $71.8 \%$, compared with that in the previously proposed core increasing the magnetic flux density to heat SFMPs by $20 \%$. However, the total iron loss in the newly designed core shape of IH coils is $1153 \mathrm{~W}$. This is not small. Thus, cooling of the newly designed E-shaped cores is necessary for the continuous and stable operations of the proposed SFMPs detector. For cooling the newly designed E-shaped cores, Fluorinert which is a brand name for the electronics coolant liquids sold commercially by $3 \mathrm{M} \mathrm{Co.,} \mathrm{Ltd.} \mathrm{is} \mathrm{used} \mathrm{to} \mathrm{cool} \mathrm{the} \mathrm{E-shaped} \mathrm{cores} \mathrm{to}$

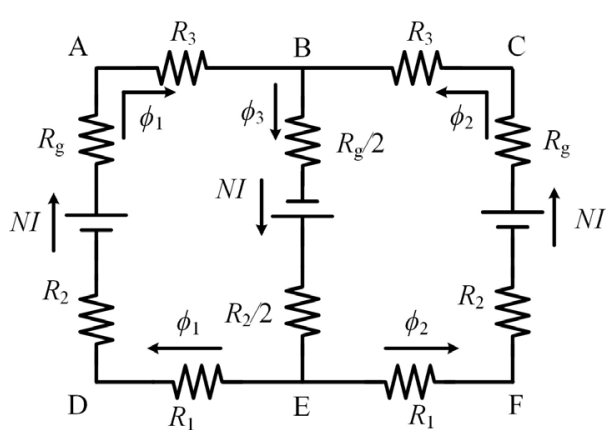

(b) 


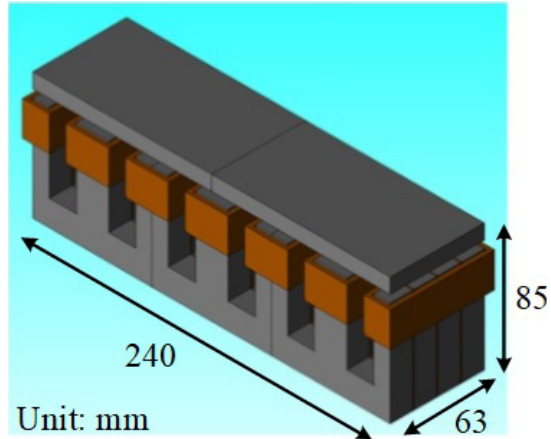

(a)

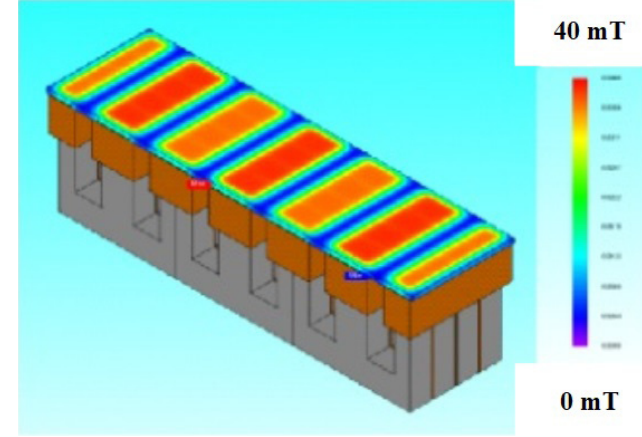

(b)

Fig. 10. Magnetic field analysis results of the magnetic flux density near the core gap modeled with JMAG software. (a) FEM model. (b) Magnetic field analysis result

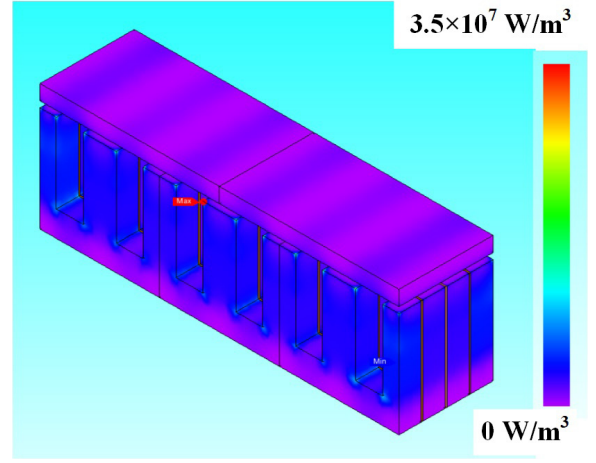

Fig. 11. Iron loss density distribution in newly proposed IH cores using JSOL JMAG software

Table 1. Comparisons of iron losses

\begin{tabular}{c|c|c}
\hline & $\begin{array}{c}\text { Previously proposed } \\
\text { IH cores }\end{array}$ & $\begin{array}{c}\text { Newly proposed } \\
\text { IH cores }\end{array}$ \\
\hline Iron loss W & 4088 & 1153 \\
\hline \hline Joule loss W & 2686 & 702 \\
\hline Hysteresis loss W & 1402 & 451 \\
\hline
\end{tabular}

avoid core cracking. Reducing the iron loss in the newly designed core shape of IH coils and cooling system of E-shaped core can avoid the temperature-rise in the core. This means that core cracking, which often occurred in (11) and (12), is also avoided with the new core design. The stable and continuous operation of the high-frequency SiC-MOSFETs based SFMPs detector with the newly designed core shape of IH coils is achieved.

Figure 12 shows the thermal analysis results of the newly proposed IH coils using JSOL JMAG software. Figure 12(a) shows the FEM model. The heatsink is used for cooling the I-shaped core. Figure 12(b) shows the average value of temperature of E-shaped, I-shaped, and heatsink of Fig. 12(a). The temperature of these cores and heatsink are saturated within one hour. As described before, Fluorinert (3M Co., LTD.) is used for continuous cooling of the E-shaped core in the experimental setup. Thus, cracking of E-shaped cores can be avoided in the experimental results described later. The temperature of the I-shaped cores with heatsink is $30^{\circ} \mathrm{C}$. This lower temperature can avoided the core cracking. Figure 13 also shows the temperature of the newly proposed IH cores and heatsink using JSOL JMAG software after the temperature of these cores and heatsink are saturated. In
Fig. 13, the temperature of I-shaped core is $30^{\circ} \mathrm{C}$. Thus, the heatsink is effective for cooling of the I-shaped core. Figures 12 and 13 demonstrate that the stable operations of the $400 \mathrm{kHz}$ SiC-MOSFETs high-frequency-inverter-based SFMP detector with the newly designed core shape of IH coils are achieved. This will be demonstrated in experimental results.

\section{Experimental Results of SFMP Heating}

A prototype experimental model of the high-frequency SiC-MOSFETs-based SFMP detector with the new core shape for IH coils is constructed and tested. Figure 14 shows a power circuit diagram of the SiC-MOSFETs highfrequency inverter, which supplies the high-frequency current to the IH coils, where the C2M0025120D (CREE Co., Ltd.) is used. Figure 15 shows a picture of the newly designed core shape of IH coils, which is used in the prototype constructed experimental model of Fig. 14. Table 2 shows circuit constants for Fig. 14. Three E-shaped cores are connected in series as shown in Fig. 15. $R_{\mathrm{o}}$ and $L_{\mathrm{o}}$ are the total equivalent resistor and inductor of the IH coils, where there are seven windings with three E-shaped cores. The bypass capacitor $C_{1}$ and $C_{2}$ of $1.12 \mu \mathrm{F}$ are connected to each leg of SiC-MOSFETs high-frequency inverter for suppressing the surge voltage, which is induced by the parasitic inductance in the dc-bus line in Fig. 14. There is a possibility of breakdown of SiC-MOSFETs by the surge voltage. Actually, SiCMOSFETs inverter for SFMPs detector are used in series resonance. However, bypass capacitor are necessary to protect from surge voltage. Litz wires are used for those windings. Enameled Litz wire are used in the newly constructed IH coils. The diameter of each wire is $0.05 \mathrm{~mm}$. A number of twists is 1800 . The breakdown voltage is $950 \mathrm{Vrms}$. A resonant capacitor $C_{\mathrm{o}}$ is connected in series to the IH coils. To avoid the cracking of the newly designed E-shaped cores caused by a spark between the windings and E-shaped cores, self-fusion silicon rubber tape (Nitto Denko Co., Ltd.) is wrapped around the Litz wires. The self-fusion silicon rubber tape has a thickness of $5 \mathrm{~mm}$, a heat-resistance temperature of $200^{\circ} \mathrm{C}$, and a breakdown voltage of $56.6 \mathrm{kV} / \mathrm{mm}$. For the SFMPs, 0.3-mm diameter stainless-steel balls (SUS304) are used.

Figure 16 shows comparisons between simulation and experimental results of the magnetic flux density at the height of 


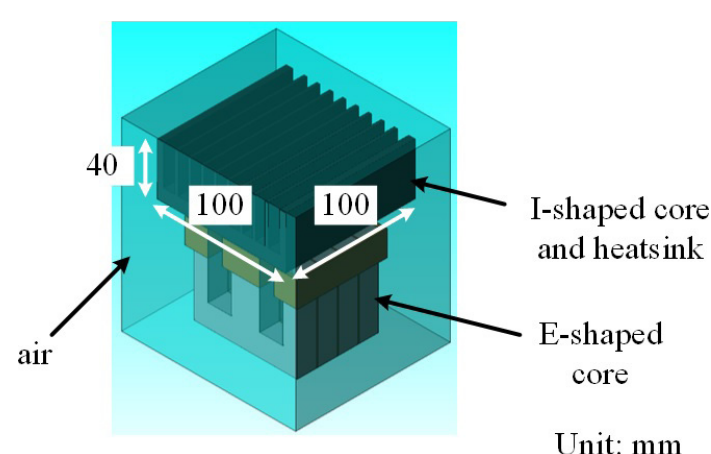

(a)

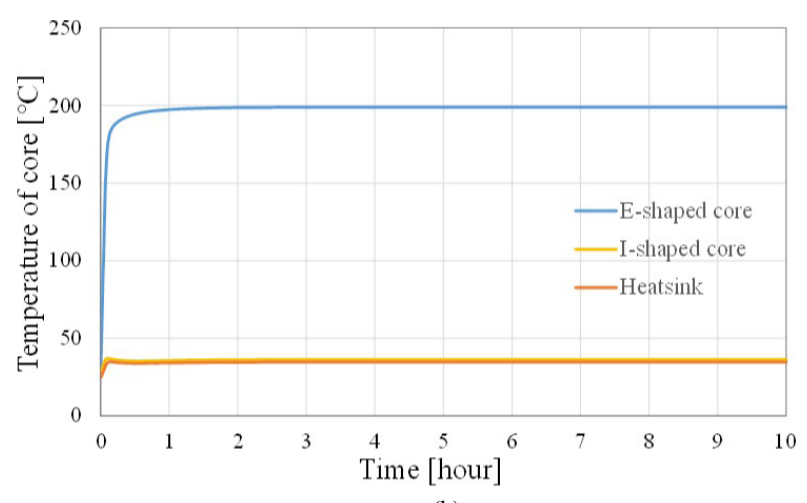

(b)

Fig. 12. Thermal analysis of the newly proposed IH coils using JSOL JMAG software. (a) FEM model. (b) Thermal analysis of the newly proposed IH coils

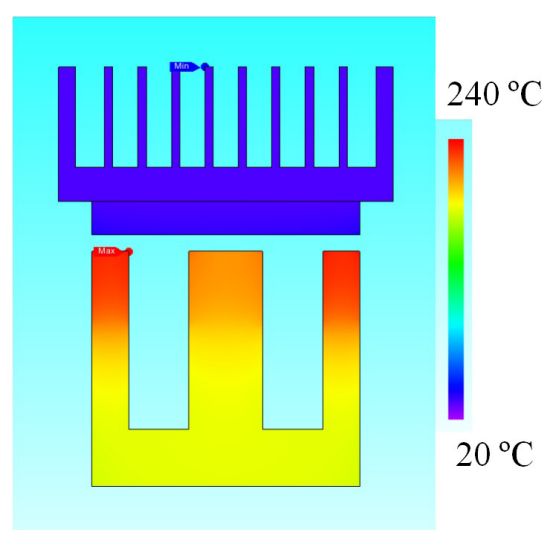

Fig. 13. Temperature of the newly proposed IH cores and heatsink using JSOL JMAG software

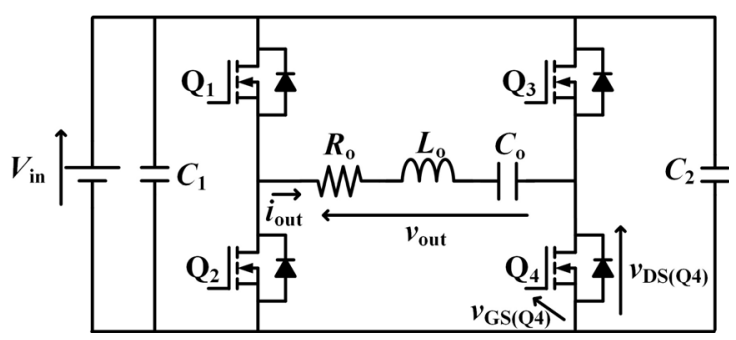

Fig. 14. Circuit diagram of SiC-MOSFETs full-bridge inverter

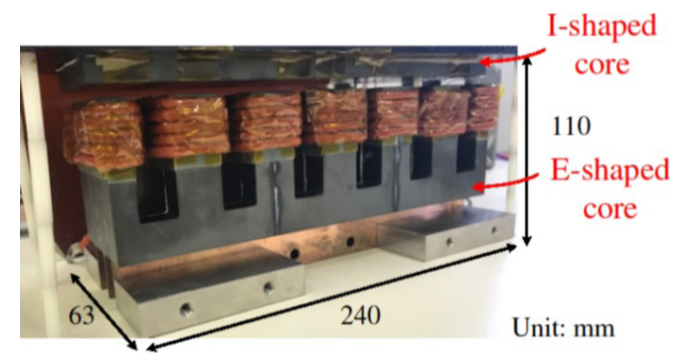

Fig. 15. Prototype of newly proposed IH coils

$3 \mathrm{~mm}$ from upper-surface of the E-shaped cores. Figure 16(a) shows the measurement points of the magnetic flux density on the upper-surface of the E-shaped cores. Figure 16(b) shows the measurement points in the experimental results of Fig. 16(a). Note that the RMS value of the coil current in the simulation results of Fig. 10 is 30 Arms, where the magnetic
Table 2. Circuit constants for Fig. 14

\begin{tabular}{c|c|c}
\hline Item & Symbol & Value \\
\hline \hline Input voltage & $V_{\text {in }}$ & $180 \mathrm{Vdc}$ \\
\hline Coil current & $I_{\text {out }}$ & $30 \mathrm{Arms}$ \\
\hline Switching frequency & $f_{\mathrm{SW}}$ & $400 \mathrm{kHz}$ \\
\hline Bypass capacitance & $C_{1}, C_{2}$ & $1.12 \mu \mathrm{F}$ \\
\hline Equivalent resistance of IH coils & $R_{\mathrm{o}}$ & $4 \Omega$ \\
\hline Inductance of IH coils & $L_{\mathrm{o}}$ & $120.2 \mu \mathrm{H}$ \\
\hline Resonant capacitor & $C_{\mathrm{o}}$ & $1.3 \mathrm{nF}$ \\
\hline Exposure time & $t$ & $5 \mathrm{~s}$ \\
\hline \multicolumn{2}{|c}{}
\end{tabular}

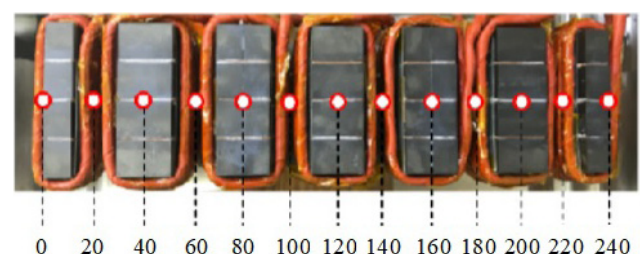

(a)

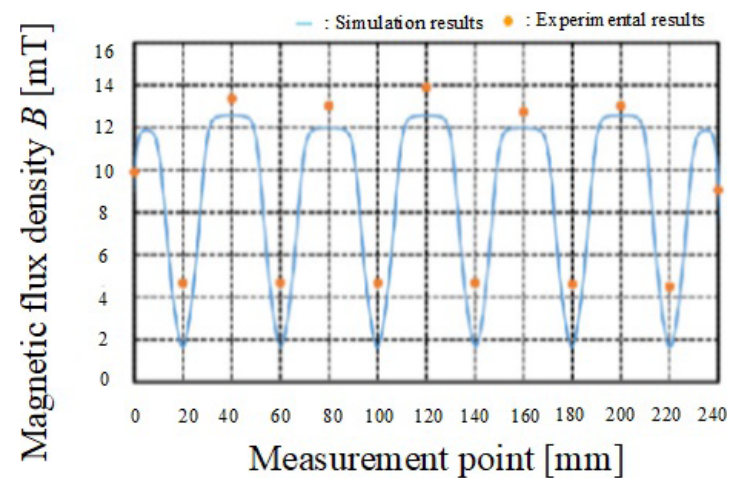

(b)

Fig. 16. Simulation results of the magnetic flux density at the height of $3 \mathrm{~mm}$ from upper-surface of the E-shaped cores. (a) Measurement points of magnetic flux density using search coil. (b) Simulated and experimental results of magnetic flux density

flux density in the core gap is $36 \mathrm{mT}$. However, the RMS value of the coil current is 10 Arms, its frequency is $400 \mathrm{kHz}$, and core material is PC40 (TDK Co., Ltd.), in Fig. 16. A search coil with the enameled copper wire was used to measure the magnetic flux density in Fig. 16. The diameter of the search coil is $6.5 \mathrm{~mm}$ and the turn-number is three. When the RMS value of the coil current was 30 Arms that equals that in Fig. 10, the used search coil was molten because of the 


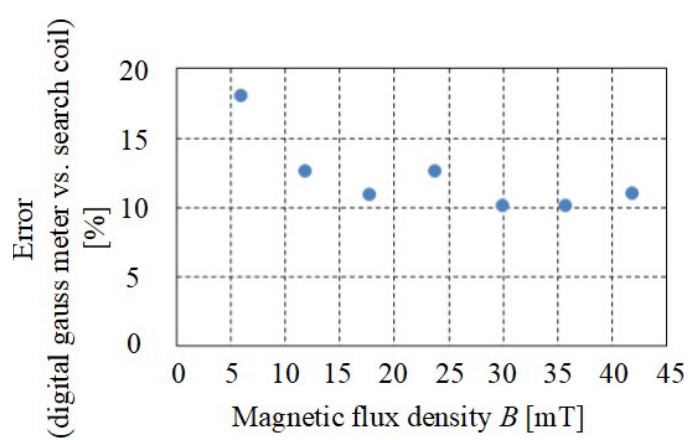

Fig. 17. Error between digital gauss meter and prototype search coil. (frequency of magnetic flux is $18.01 \mathrm{kHz})$

high magnetic density of $36 \mathrm{mT}$ with the high frequency coil current of $400 \mathrm{kHz}$. This demonstrates that the comparisons between simulation and experimental results of the magnetic flux density at the height of $3 \mathrm{~mm}$ from the upper-surface of the E-shaped cores with the current coil of 30 Arms is impossible. Thus, the RMS value of the coil current is 10 Arms in simulation and experimental results in Fig. 16. The blue colored line shows the simulation results using JSOL JMAG software. The orange colored dots show the experimental results using the search coil. The maximum errors between the simulation results and the experimental results are less than $15 \%$. The experimental results of the magnetic flux density measurement using the search coil well agree with the simulation results using JSOL JMAG software. From the simulation results of Fig. 10, the authors have concluded that the magnetic flux density is approximately $36 \mathrm{mT}$ in the core gap between E-and I-shaped cores if the RMS value of the coil current is 30 Arms.

The detection error of the used search coil is discussed, here. No digital gauss meters for the high-frequency alternative magnetic flux, for example for $400 \mathrm{kHz}$, is on commercial. A commercial digital gauss-meter, which is 5170 (TOYO Corp.), can measure the magnetic flux density from DC to $20 \mathrm{kHz}$. Using this digital gauss meter, the detection errors of the search coil are evaluated. Figure 17 shows the detection errors between the search coil and the commercial digital gauss meter, where the frequency of the magnetic flux is $18.01 \mathrm{kHz}$. The detection error is about $18 \%$ for $5 \mathrm{mT}$. The detection errors are around $13 \%$ when the magnetic flux density is higher than over $10 \mathrm{mT}$. It is concluded the differences between the simulation results and experimental results are caused by the detection errors of the search coil. As described above, the diameter of the search coil is $6.5 \mathrm{~mm}$ and the turn-number is three. The detection errors increase at the measurement points $20 \mathrm{~mm}, 60 \mathrm{~mm}, 100 \mathrm{~mm} 140 \mathrm{~mm}$, $180 \mathrm{~mm}$, and $220 \mathrm{~mm}$. These detection errors are also caused by the diameter of $6.5 \mathrm{~mm}$ in the search coil. In the simulation results, there is the same maximum value at the $40 \mathrm{~mm}$, $120 \mathrm{~mm}$, and $200 \mathrm{~mm}$ measurement-points. There also is the same value at the $0 \mathrm{~mm}, 80 \mathrm{~mm}, 160 \mathrm{~mm}$, and $240 \mathrm{~mm}$ measurement-points. This is an important issue for further study. Theoretical discussions confirmed with the digital computer simulation will be reported in another article.

Figure 18 shows the experimental waveforms of SiCMOSFETs high-frequency inverter shown in Fig. 14. Figure 18(a) shows the gate-source and drain-source voltage

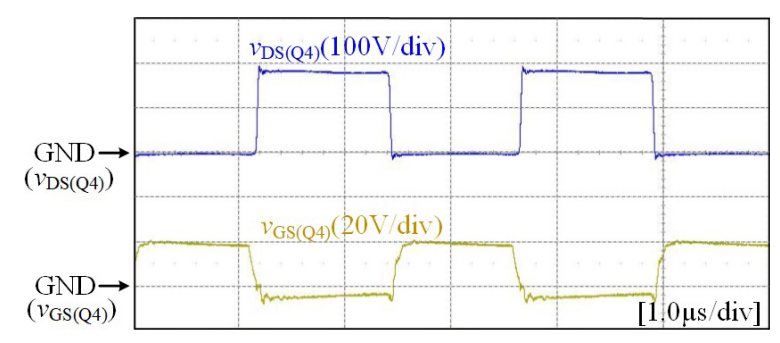

(a)

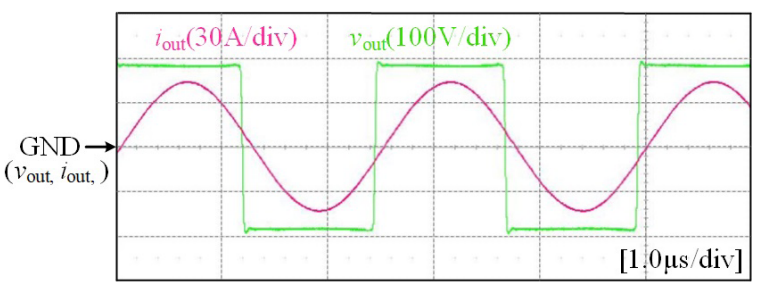

(b)

Fig. 18. Experimental waveforms of SiC-MOSFETs high-frequency inverter. (a) Gate-source voltage and drain-source voltage waveforms for $Q_{4}$. (b) Output voltage and current waveforms

waveforms for $Q_{4}$ of Fig. 14. Figure 18(b) shows the output voltage and current waveforms for Fig. 14, where the RMS value of output current is 30.5 Arms and frequency is $403.5 \mathrm{kHz}$. From these experimental waveforms, the stable operation of $400 \mathrm{kHz}$ SiC-MOSFETs high-frequency inverter is achieved.

Figure 19 shows the locations of the SFMPs and experimental results. Figure 19(a) shows the locations of $0.3-\mathrm{mm}$ diameter stainless-steel balls that used as model SFMPs. The gap between the E-shaped core and I-shaped core is $5 \mathrm{~mm}$. The film is placed in the gap between the E-shaped core and I-shaped core, as shown in Fig. 15 to heat the model SFMPs. After an exposure of the magnetic flux with the coil currents of $30 \mathrm{Arms}$ for $5 \mathrm{~s}$, a temperature rise of the stainless-steel balls is observed using a thermographic camera (R300SR, Nippon Avionics Co. LTD.). Figure 19(b) shows the observed SFMPs using the thermographic camera. The temperature difference $\Delta T_{\max }$ between the heated stainless-steel balls and room temperature is $6.6^{\circ} \mathrm{C}$. From the experimental results shown in Fig. 19, the authors conclude that the high-frequency SiC-MOSFETs-based SFMP detector with the newly designed core shape of IH coils is useful for industrial continuous production lines of HPCFs.

\section{Conclusion}

In this paper, a core shape of IH coils with a $400 \mathrm{kHz}$ SiC-MOSFETs high-frequency-inverter-based SFMP detector, which can reduce iron loss, has newly been designed for a stable and continuous operations to detect SFMPs. A magnetic field analysis has been performed to confirm the validity and high practicability of the newly designed core shape of IH coils using JSOL JMAG software. The magnetic field analysis results have demonstrated that iron loss in the newly designed core shape is reduced by $71.8 \%$, compared with that in the previously designed core increasing the magnetic flux density to heat the SFMPs by $20 \%$. Reducing iron loss in the newly designed core shape of IH coils results in a stable and continuous operation owing to lowered 


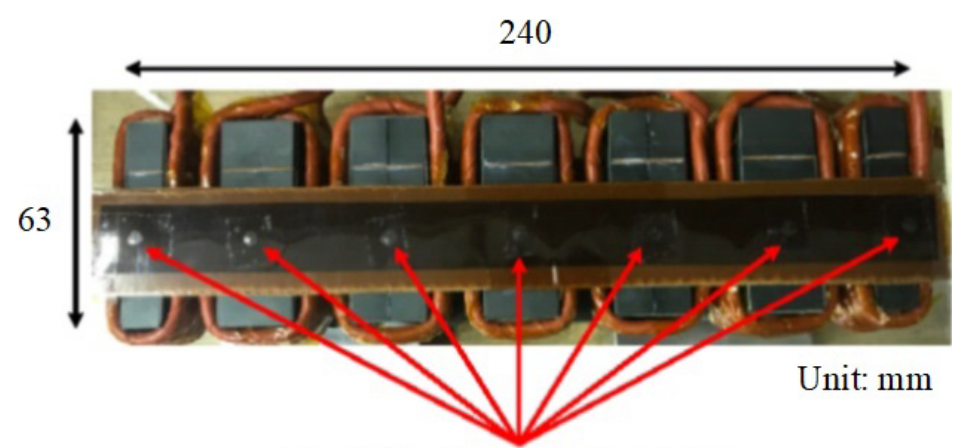

Small-foreign-metal particles

(a)

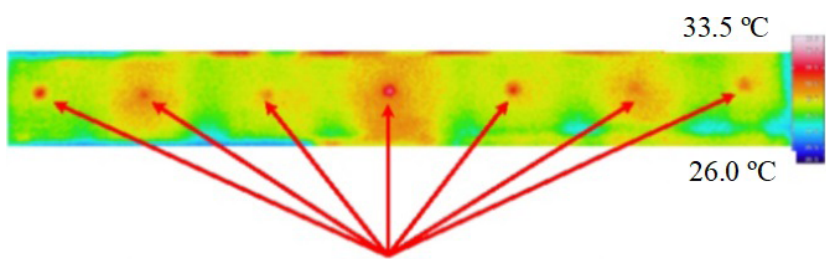

Observed small-foreign-metal particles

(b)

Fig. 19. Prototype model of newly proposed IH coil. (a) Top view of IH cores. (b) Thermal image using thermographic camera

thermal stress damage. A prototype experimental model of the high-frequency SiC-MOSFETs inverter-based SFMP detector with the newly designed core shape of IH coils has been constructed and tested. Experimental results have demonstrated that a $400 \mathrm{kHz}$ SiC-MOSFETs high-frequency inverter-based SFMP detector with the newly designed core shape of IH coils can heat $0.3-\mathrm{mm}$ diameter SFMPs, and the heated SFMPs can be observed using a thermographic camera. Based on the experimental results, the authors have concluded that the $400 \mathrm{kHz}$ SiC-MOSFETs high-frequency inverter-based SFMP detector with the newly designed core shape of IH coils can be applied to HPCF production lines.

Finally, this paper is an improved and revised version of the conference paper (13). The authors would like to express their gratitude the audience for their valuable discussions at the International Power Electronics Conference (IPEC 2018). This work was supported by Council for Science, Technology and Innovation (CSTI), Cross-ministerial Strategic Innovation Promotion Program (SIP), "Next-generation power electronics" (funding agency: NEDO).

\section{References}

( 1 ) D. Capozzo, S. Fleming, B. Foley, and M. Macri: "Lithium ion battery safety", Worcester Polytechnic Institute, pp.1-169 (2006)

( 2 ) M. Arakawa and M. Ichimura: "Latest safety test technologies of lithium-ion batteries and construction of battery safety evaluation site", NTT Facilities Research Institute, Annual Report No.20, pp.1-7 (2009)

( 3 ) M. Ikeda, A. Mase, and K. Uchino: "Small-conductive-particle detection with a microwave resonant cavity", IEEJ Trans. on Ind. Appl., Vol.132, No.8, pp.788-793 (2011)

( 4 ) T. Nakai, Y. Miura, and K. Ishiyama: "Method of detection a small magnetic particle by using a sensitive thin-film sensor with application of strong magnetic field in the substrates normal direction", IEEJ Annu. Meet., p.137 (2013)
( 5 ) S. Tanaka, T. Akai, and Y. Kitamura: "Two-channel HTS SQUID gradiometer system for detection metallic contaminants in lithium-ion battery", IEEE Transactions on Applied Superconductivity, Vol.21, No.3, pp.424-427 (2011)

( 6 ) S. Tanaka, T. Akai, Y. Hatsukabe, T. Ohtani, Y. Ikeda, S. Suzuki, and K.Tanabe: "High Tc SQUID detection system for metallic contaminants in lithium ion battery", IEEE Transactions on Magnetic, Vol.45, No.10, pp.4510-4513 (2009)

( 7 ) Y. Hatsukabe, T. Abe, S. Tsunaki, M. Yamamoto, H. Murata, and S. Tanaka: "Application of ultra-low field HTS SQUID NMR/MRI to contaminant detection in food", IEEE Transactions on Applied Superconductivity, Vol.23, No.3 (2011)

( 8 ) T. Ohtani, Y. Narita, S. Tanaka, S. Ariyoshi, and S. Suzuki: "Development of three channel SQUIDs contaminant detector for food inspection", IEEE Int. Magnetic Conf. (INTERMAG), Beijing, China (2015)

( 9 ) S. Kurachi, N. Yamamoto, H. Yamada, T. Tanaka, E. Hiraki, Y. Yamada, T. Nagao, Y. Miyake, and Y. Noda: "High-frequency induction heating for tiny foreign metals", in Proc. of 9th International Conference on Power Electronics-ECCE Asia (ICPE 2015-ECCE Asia), Seoul, South Korea, pp.2203-2208 (2015)

(10) T. Shijo, S. Kurachi, Y. Noda, H. Yamada, and T. Tanaka: "A 400 kHz SiCMOSFETs high-frequency inverter for small-foreign-metal particle detection", in Proc. of IEEE Power Electronics Society Southern Power Electron. Conf. (SPEC), New Zealand, Auckland, S27-423 (2016)

(11) T. Shijo, S. Kurachi, Yuki Uchino, Y. Noda, H. Yamada, and T. Tanaka: "High-frequency induction heating for small-foreign-metal particles using SiC-MOSFETs inverter", in Proc. of IEEE International Future Energy Electronics Conference (IFEEC 2017-ECCE-Asia), Kaohsiung, Taiwan, pp.615620 (2017)

(12) T. Shijo, S. Kurachi, Yuki Uchino, Y. Noda, H. Yamada, and T. Tanaka: "High-frequency induction heating for small-foreign-metal particles detection using $400 \mathrm{kHz}$ SiC-MOSFETs inverter", in Proc. of IEEE Energy Conversion Congress and Expo. (ECCE), Cincinnati, Ohio, pp.5133-5138 (2017)

(13) T. Shijo, Y. Uchino, Y. Noda, H. Yamada, and T. Tanaka: "Iron loss reduction in the cores of induction heating coils for small-foreign-metal particle detector with a 400-kHz SiC-MOSFETs high-frequency inverter", in Proc. of International Power Electronics Conference (IPEC 2018-ECCE-Asia), Niigata, Japan, pp.324-328 (2018) 
Takuya Shijo (Student Member) received the B.E. and M.E. degrees

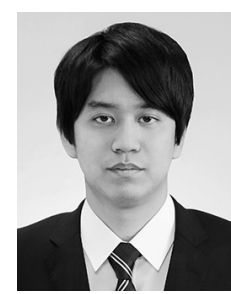
from Yamaguchi University in 2016 and 2017, respectively. He is currently working toward the Ph.D degree in engineering. He is engaged in research on high-frequency induction heating for small-foreignmetal particles detection using SiC-MOSFETs inverter. He is a student member of the IEEE.

Yuki Uchino (Student Member) received the B.E. and M.E. degrees

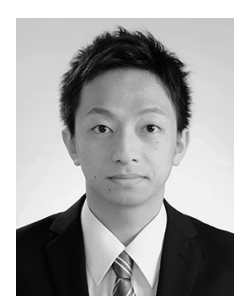
from Yamaguchi University in 2017 and 2019, respectively. In 2019, he has joined TOSHIBA CORPORATION. He was engaged in research on highfrequency induction heating for small-foreign-metal particles detection using SiC-MOSFETs inverter

Yujiro Noda (Member) graduated from National Fisheries University

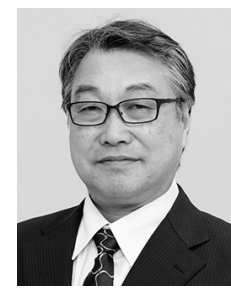
in 1982. He joined the Digital Equipment Corporation (DEC) in 1982. Since 1986, he has been with the Ube Industries. He is currently working toward the Ph.D. degree in engineering at Yamaguchi University. $\mathrm{He}$ is engaged in research on high-frequency induction heating for small-foreign-metal particles detection using SiC-MOSFETs inverter.
Hiroaki Yamada (Member) received the M.E. degree from Shimane

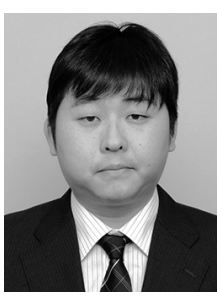
University in 2004. In 2007, he received the Doctor of Engineering from Yamaguchi University. From 2007 to 2010, he was a Lecturer at Kushiro National College of Technology. From 2010 to 2014, he was an Assistant Professor at Kyushu Institute of Technology. From 2014 to 2018, he was a Lecture in the Department of Electrical and Electronic Engineering at Yamaguchi University. He is currently an Associate Professor. His research interests include power conversion systems for LED driver and wind power generation system. Dr. Yamada is a member of the IEEE.

Toshihiko Tanaka (Fellow) received the M.E. degree from Na-

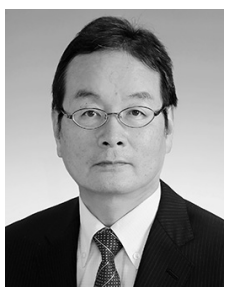
gaoka University of Technology in 1984. In 1995, he received the Ph.D. degree in Engineering from Okayama University, Japan. He joined Toyo Denki Mfg. Co. in 1984. From 1991 to 1997, he was an Assistant Professor at the Polytechnic University of Japan. From 1997 to 2004, he was an Associate Professor at Shimane University. Since 2004, he has been a Professor in the Department of Electrical and Electronic Engineering at Yamaguchi University. His research interests are on harmonics generated by static power converters and their compensation. Dr. Tanaka is a member of the IEEE. 